\title{
Processing and Properties of Bar-Shaped Single-Seeded and Multi-Seeded YBCO Bulk Superconductors by a Top-Seeded Melt Growth Technique
}

\author{
Abdulmajeed Almalki $^{1}$ - Devendra K. Namburi ${ }^{2}$ (i) - Mazen Ba-Abbad ${ }^{1}$. \\ Anthony R. Dennis $^{2}$ - K. Y. Huang ${ }^{2}$ - Abdul-Aziz Almutairi ${ }^{1}$ - J. H. Durrell ${ }^{2}$. \\ David A. Cardwell ${ }^{2}$
}

Received: 14 November 2016 / Accepted: 29 November 2016 / Published online: 30 December 2016

(C) The Author(s) 2016. This article is published with open access at Springerlink.com

\begin{abstract}
The fabrication of (RE)-Ba-Cu-O bulk superconductors, where RE is a rare-earth element such as $\mathrm{Y}, \mathrm{Gd}$ and $\mathrm{Sm}$, is both time consuming and expensive due to the complexity of the melt process and the slow growth rate of large, single grains. In this study, different approaches to the fabrication of bar-shaped, bulk YBCO superconductors are investigated and compared using single- and multipleseeding techniques via top-seeded melt growth (TSMG). Both the microstructural and superconducting properties of the bulk samples are investigated, including trapped field, critical current density, critical temperature and levitation force. The results of this study indicate that, in general, the superconducting properties of YBCO fabricated by a singleseeded process are significantly better than those of samples fabricated by a four-seeded process for non-bridge seeds. The differences between the samples are less pronounced in the levitation force measurements, however. In this paper, we attempt to explain the reasons for the similarities and differences observed between bulk samples fabricated by the different single- and multi-seeded processes.
\end{abstract}

Keywords YBCO - Bulk superconductors ·

Multi-seeding · Current density · Levitation force .

Trapped field

Devendra K. Namburi

dkn23@cam.ac.uk; ndevendra@gmail.com

1 Water and Energy Institute, King Abdulaziz City for Science and Technology, Riyadh, Saudi Arabia

2 Bulk Superconductivity Group, Department of Engineering, University of Cambridge, Cambridge, UK

\section{Introduction}

Y-Ba-Cu-O (YBCO), and the related cuprates containing different rare-earth (RE) elements, is one of the so-called type II, high-temperature superconducting oxide ceramic materials with a critical temperature of 90-92 K $[1,2]$. YBCO in bulk form can be machined relatively easily at room temperature and is therefore a good candidate for practical applications, such as trapped field magnets, flywheel energy storage and non-contact bearings for frictionfree motors [1-3]. The critical current density $\left(J_{\mathrm{c}}\right)$ and the trapped magnetic flux density $\left(B_{\mathrm{t}}\right)$ are the two most important properties of YBCO bulk superconductors from an application perspective $[4,5]$. In addition, the trapped magnetic flux density is proportional to the critical current density $\left(J_{\mathrm{c}}\right)$ and the diameter of the supercurrent loop $(d)$, i.e. $B_{\mathrm{t}} \propto J_{\mathrm{c}} \times d$, which is an important figure of merit for bulk superconductors [5].

Various growth techniques, including melt-textured growth (MTG), melt powder melt growth (MPMG), powder melting process (PMP) and top-seeded melt growth (TSMG) [6-9], have been developed over the past 25 years in order to fabricate YBCO bulk superconductors in large, single grain form. Of these, the TSMG technique has been studied the most extensively and has emerged as a reliable process for fabricating large (RE)BCO bulk, single grains.

Sample microstructure plays a key role in determining the flux pinning strength in type II superconductors. The presence of non-superconducting phases in the form of defects, ideally of the order of magnitude in size of the coherence length (1-3 nm at around $20 \mathrm{~K})$, impedes the motion of flux vortices and, as a result, increases the trapped field $\left(B_{\mathrm{t}}\right)$. It has been found that a reduction in the size of $\mathrm{RE}_{2} \mathrm{BaCuO}_{5}$ '(RE)-211' secondary-phase particles 
embedded within the superconducting (RE) $\mathrm{Ba}_{2} \mathrm{Cu}_{3} \mathrm{O}_{7-y}$ '(RE)-123'-phase matrix that occur naturally as a result of the melt growth process improves the critical current density of (RE)BCO [10-12]. The concentration of Y-211 particles in YBCO is known to vary considerably from the region close to the seed crystal to the edge/bottom of the sample [13]. This is an unavoidable effect, which occurs due to particle pushing effects associated with the $\mathrm{YBa}_{2} \mathrm{Cu}_{3} \mathrm{O}_{7-x}$ (Y-123) growth front. Recently, composition grading techniques were employed to minimize inhomogeneities in the Y-211 concentration throughout the volume of the sample, thereby achieving greater uniformity in its superconducting properties $[9,10,14]$.

The TSMG technique involves the incongruent melting of the Y-123 phase above its peritectic temperature $\left(T_{\mathrm{p}}\right)(\sim$ $1005^{\circ} \mathrm{C}$ in air), which decomposes to Y-211 (solid) and a liquid phase comprising $\mathrm{BaCuO}_{2}$ and $\mathrm{CuO}$. A seed crystal (whose melting temperature is above the $T_{\mathrm{p}}$ of the seeded bulk, but with similar crystal lattice parameters and having phase stability with the melt) is employed for seeding and subsequent growth of YBCO into a single grain. The growth rate of the Y-123 phase is of the order of $0.2-0.4 \mathrm{~mm} / \mathrm{h}[12$, 13], which limits practically the ultimate size of the final single grain. Additionally, it has been reported previously that large samples exhibit increasing crystallographic misorientation of the Y-123 phase with increasing distance from the seed [14]. As a result, it is not easy to fabricate (RE)BCO single grains reliably in sizes of diameter larger than $\sim 40$ $\mathrm{mm}$. The increasing drive towards applications requires the development of methods to produce larger (RE)BCO bulk samples both reliably and economically.

In this context, multi-seeding is one approach that can be used to fabricate large-sized (RE)BCO in quasi-single grain form and reduce simultaneously the processing time. In particular, magnetic levitation forces and stiffness ratios for both multi- and single-seeded bulk superconductors have been observed to increase with increasing cooling times [15]. Seeding techniques have been employed to grow YBCO single grains [16] with both $0^{\circ}-0^{\circ}$ and $45^{\circ}-45^{\circ}$ orientations of the seed. Here, for instance, $0^{\circ}-0^{\circ}$ represents the angles between the direction of the long axis of a bridge seed and the $a$ direction [corresponding to the (100) direction for the tetragonal YBCO phase] of the two seeds, respectively $[16,17]$. The bridge technique has enabled significant improvement in the alignment of the seeds and has eliminated the sharp decrease in trapped field observed at the centre of YBCO bulk superconductors fabricated using independent (i.e. non-bridge) seeds. A detailed comparison between melt processes using $0^{\circ}-0^{\circ}$ and $45^{\circ}-45^{\circ}$ bridge seeds has been reported by Shi et al. [17]. This clarified that the $45^{\circ}-45^{\circ}$ bridge seeding results in cleaner grain boundaries in comparison with $0^{\circ}-0^{\circ}$ bridge seeds.
GdBCO and YBCO bulk superconductors have also been fabricated via a multi-seeding technique employing welloriented NdBCO thin film seeds $[18,19]$. The use of multi-seeds arranged asymmetrically with 110/110 orientation resulted in a promising approach for enlarging the domain size of GdBCO bulk samples. However, the NdBCO thin film seeds used to grow YBCO were damaged partly during processing due to contact with the liquid-phase components at elevated temperature $[20,21]$, which resulted in the undesirable growth of Y-123 grains with a 001/103 orientation. This partial melting of seed crystals can be avoided to a certain extent by invoking either two-step cooling during the melt process [22] or by employing the buffer technique [23-25]. Also, it has been reported that the YBCO single grains grow much larger if the cooling time at the growth temperature is increased $[12,13]$.

The levitation force generated between the bulk sample and the load is the most critical parameter for the successful development of systems such as flywheels and contactless bearings. In this paper, we compare the key applied properties of single-seeded and multi-seeded YBCO bar-shaped samples in order to determine whether multi-seeding represents an effective route for the production of larger samples, as required in several applications.

\section{Experimental Details}

Single-seeded and four-seeded Y-Ba-Cu-O (YBCO) bulk superconductors were fabricated by the TSMG technique using Mg-doped NdBCO generic seeds [26] from a precursor powder of composition 30 wt $\%$ of Y-211 +70

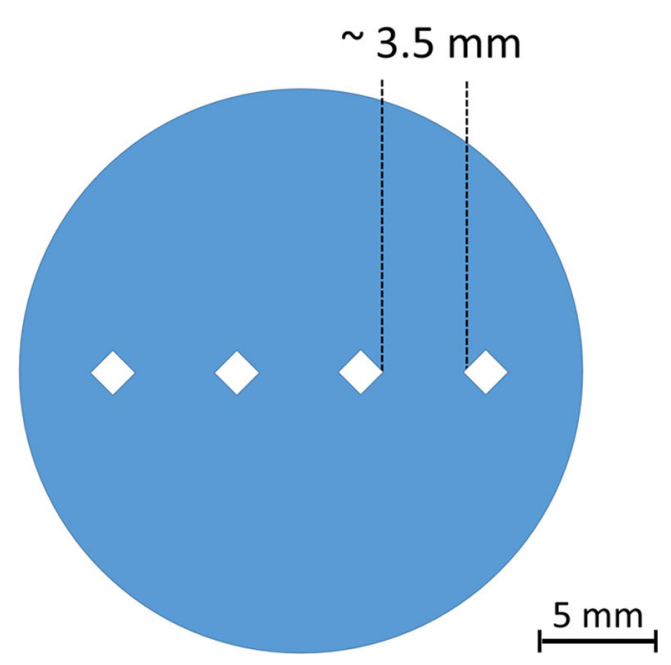

Fig. 1 Schematic diagram showing the orientation and spacing of the $\mathrm{Mg}$-doped NdBCO generic seeds on the top surface of the green-phase sample prior to melt processing 


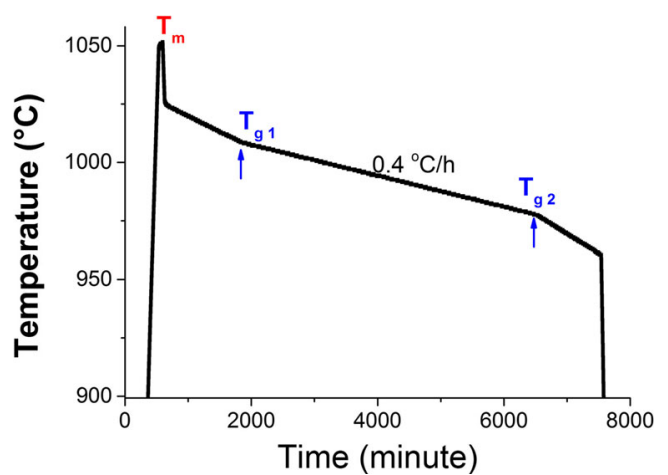

Fig. 2 Temperature-time profile for processing YBCO bulk samples by the top-seeded melt growth technique

wt $\%$ of Y-123 +1 wt $\%$ of $\mathrm{CeO}_{2}$. The resultant powder mixture was pressed uniaxially (25 MPa) into a cylindrical pellet of diameter $25 \mathrm{~mm}$. The single-seeded sample was grown using a cleaved $\mathrm{Mg}$-doped $\mathrm{NdBCO}$ generic seed crystal placed at the centre of the pressed green pellet. The

a
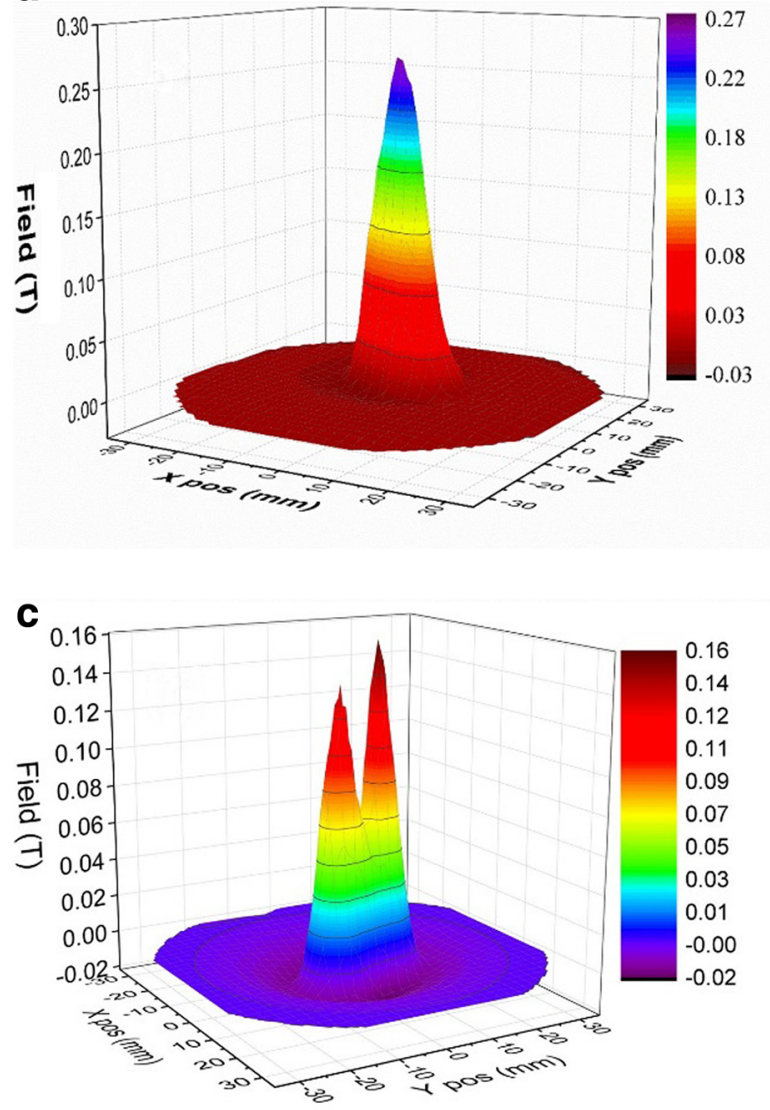

generic seed crystal was chosen for this application since it is known to have a significantly higher melting temperature than the composition of the target single grain and can hence overcome the problems of partial melting associated with seed crystal due to its potential contact with the liquid phase during processing [26]. Four seeds prepared as for the single-seeded sample were placed on the top surface of the green pellet. The distance between each of the two seeds was $\sim 3.5 \mathrm{~mm}$, as shown schematically in Fig. 1 .

The pressed samples with seeds were melt processed in a box furnace. They were heated initially at $875^{\circ} \mathrm{C}$ at a rate of $100{ }^{\circ} \mathrm{C} / \mathrm{h}$ and then at $1051{ }^{\circ} \mathrm{C}\left(T_{\mathrm{m}}\right)$ at a rate of $50{ }^{\circ} \mathrm{C} / \mathrm{h}$ and held at this temperature for $60 \mathrm{~min}$. They were then cooled rapidly at $1025^{\circ} \mathrm{C}$ at a rate of $75^{\circ} \mathrm{C} / \mathrm{h}$ and slowly at 1008 ${ }^{\circ} \mathrm{C}\left(T_{\mathrm{g} 1}\right)$ (cooling rate 1$)$, followed by slow cooling at 977 ${ }^{\circ} \mathrm{C}\left(T_{\mathrm{g} 2}\right)$ at the rate of $0.4{ }^{\circ} \mathrm{C} / \mathrm{h}$ (cooling rate 2$)$. Finally, the samples were furnace cooled at room temperature. This temperature profile is illustrated schematically in Fig. 2.

The fully melt-processed bulk samples were annealed in flowing oxygen at $425{ }^{\circ} \mathrm{C}$ for 10 days in order to obtain the optimally oxygenated orthorhombic Y-123 phase. The
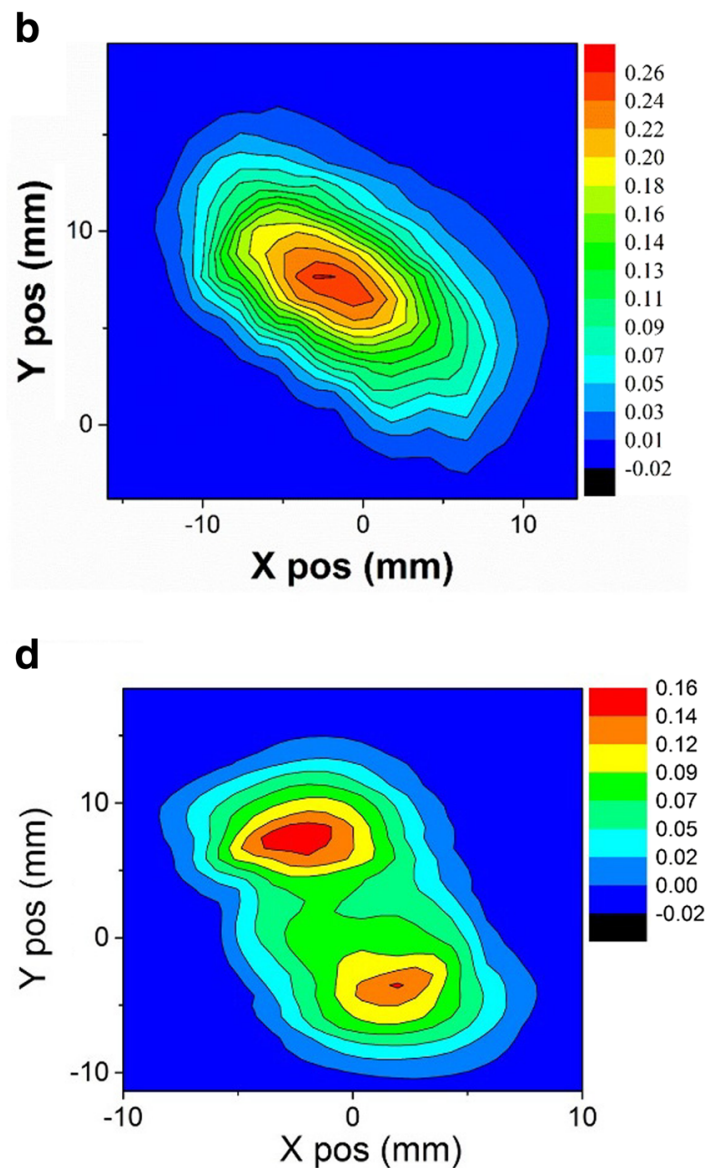

Fig. 3 Trapped field profiles (both the 3D profiles and the 2D contour maps) obtained for the single-seeded YBCO sample (a, b) and the four-seeded YBCO sample (c, d) 
melt-processed samples were cut into rectangular cross sections of $20 \mathrm{~mm} \times 8 \mathrm{~mm}$ dimensions and $10 \mathrm{~mm}$ thickness. The samples were cooled at $77 \mathrm{~K}$ in liquid nitrogen in a magnetic field of $1.4 \mathrm{~T}$ applied parallel to the $c$-axis of the sample to measure the maximum magnetic field that could be trapped at this temperature. This was measured using a handheld Hall probe, followed by field mapping using a rotating array of Hall probes at a distance of $1-1.5 \mathrm{~mm}$ from the top surface of the sample.

A stack of three rectangular $\mathrm{NdFeB}$ permanent magnets of dimensions $25 \mathrm{~mm} \times 10 \mathrm{~mm} \times 10.5 \mathrm{~mm}$ with an operating magnetic field strength of $0.4 \mathrm{~T}$ was used to measure the levitation forces produced by the samples at $77 \mathrm{~K}$. The levitation force between the magnet and the samples was measured using a load cell. The approach/recede rate of the magnet to the sample was controlled at $0.2 \mathrm{~mm} / \mathrm{s}$.

Both samples were polished in order to observe their microstructures via an optical microscope. The samples were subsequently sliced into smaller sub-specimens for further measurement of their superconducting properties. A small sub-specimen of size $2.35 \mathrm{~mm} \times 2.43 \mathrm{~mm} \times 1.8 \mathrm{~mm}$ sliced and cleaved from each single grain sample was used to measure the transition temperature $\left(T_{\mathrm{c}}\right)$ and the critical current density $\left(J_{\mathrm{c}}\right)$ at $77 \mathrm{~K}$ using a superconducting quantum interference device (SQUID) magnetometer (Quantum Design MPMS-XL). Kim's extended model [27] was used to deduce the critical current densities from the measured magnetic hysteresis loops.

\section{Results and Discussion}

Figure $3 \mathrm{a}, \mathrm{b}$ shows that the single-seeded, single grain sample exhibits a trapped field at the top surface that consists of a single peak and smooth gradient from the centre towards the sample edge. The sample trapped a field of $373 \mathrm{mT}$ (peak value) as measured at the surface of the sample using the handheld Hall probe $(260 \mathrm{mT}$ measured at a distance of $1.5 \mathrm{~mm}$ from the sample surface, as shown in Fig. 3a, b). The corresponding measured trapped field profiles for the multi-seeded sample are shown in Fig. 3c, d. Although four seeds were employed, the profile shows the presence of two peaks. The trapped field appears to decrease to a negligible value between the two seeded growth regions at the middle of the sample, suggesting that there is no connectivity between the separately seeded regions. The maximum trapped field of this sample was $253 \mathrm{mT}$ at the surface of the sample ( $150 \mathrm{mT}$ as measured at a distance of $1.5 \mathrm{~mm}$ above the sample surface), which is significantly lower than that of the single grain sample.

The levitation force-displacement curves obtained for the single-seeded and the multi-seeded YBCO samples are shown in Fig. 4. In each case, the levitation force was
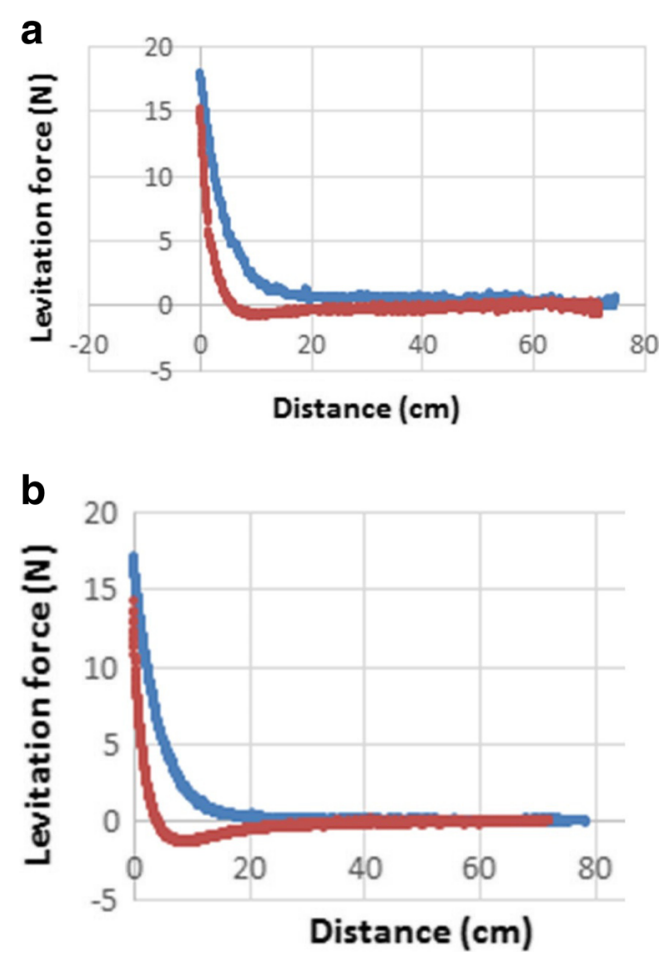

Fig. 4 Levitation force-displacement curves obtained for a singleseeded and $\mathbf{b}$ four-seeded YBCO samples

measured by moving a rectangular-shaped magnet either towards or away from the zero-field-cooled (ZFC) superconductor at $77 \mathrm{~K}$. Although the trapped field in the singleseeded sample resulted in a higher value than that obtained in the multi-seeded sample, the levitation forces obtained for both samples were very similar (peak values of $17 \mathrm{~N}$ for the multi-seeded sample and $18 \mathrm{~N}$ for the single-seed sample at $77 \mathrm{~K}$ ). The blue curve in Fig. 4 represents the situation where the magnet is approaching the superconductor, and the red curve represents the data where the magnet is moving away from after the initial approach. This suggests that the levitation produced by ZFC samples is not strictly dependent on the quality of the grain and that the levitation force is dependent more critically on the net strength and density of the flux pinning centres.

Figure 5 shows a photograph of the top surface of the four-seeded sample after polishing. It can be seen that,

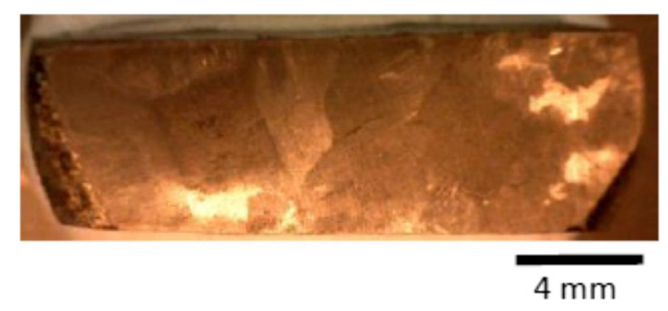

Fig. 5 Photograph of the top surface of the four-seeded YBCO sample after polishing. Growth of only two grains can be clearly seen 
Fig. 6 a Schematic diagram illustrating the centre $(C)$ and the edge $(E)$ positions from where the micrographs were recorded. Optical micrographs obtained under a magnification of $\times 100$ and $\times 1000$ from the single- and four-seeded $\mathrm{YBCO}$ samples are shown in $\mathbf{b}-\mathbf{e}$ and $\mathbf{f}-\mathbf{i}$, respectively

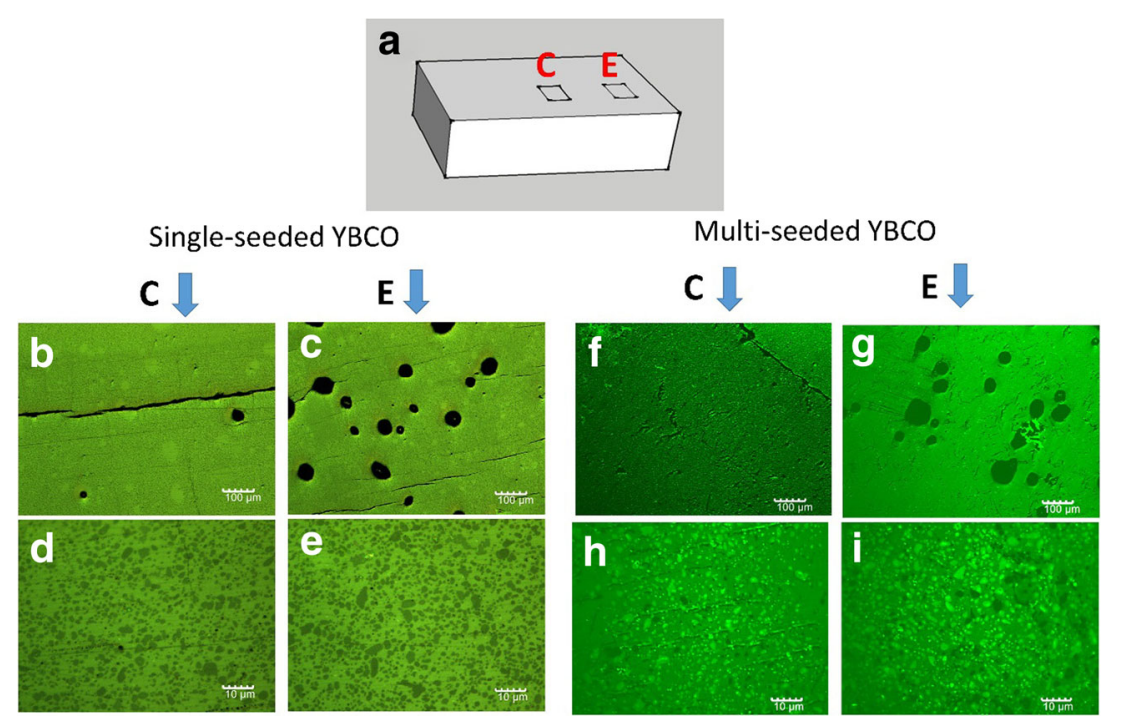

although four seeds were employed, only two grains resulted from the TSMG process. Furthermore, the two grains are not well aligned due partially to the fact that the alignment of the seeds could have changed at the elevated processing temperature. Misalignment of grains will clearly influence the trapped field ability of the bulk samples.

The microstructures of the single-seeded and four-seeded samples are illustrated in Fig. 6b-e and f-i, respectively. It was observed that the embedded Y-211 particles accumulate predominantly towards the edge of the single-seeded sample. This is attributed to the effects of particle pushing, which is common in the TSMG-processed (RE)BCO. However, this effect was observed to be less pronounced in the multi-seeded sample. In general, the images show that Y211 particles in the present samples are homogenous and well refined.

It is known that the size and distribution of the embedded Y-211 particles influences the field trapping capabilities of the bulk sample. In the single- and multi-seeded samples produced here, however, it is the size of the current loops that has an overriding effect on the peak value of trapped field of each sample. The presence of two smaller current loops observed in the multi-seeded sample limits the trapped field performance when compared to that of the single-seeded sample. In fact, this argument is well supported, given that the current loop is approximately double the size in the single-seeded sample and this is reflected in the observed values of trapped fields.

A schematic illustration of the position of the specimen in the parent single grain used to measure $T_{\mathrm{c}}$ is shown in Fig. 7. The corresponding transition temperature $\left(T_{\mathrm{c}}\right)$ data are shown in Fig. 8a, from which it can be seen that the onset of $T_{\mathrm{c}}$ of the sample is $\sim 90 \mathrm{~K}$. The fact that the sample exhibits a sharp transition indicates that it is well oxygenated and that transformation from the tetragonal to orthorhombic state is complete. Figure $8 \mathrm{~b}$ shows the field dependence of $J_{\mathrm{c}}$ at $77 \mathrm{~K}$ for the same specimen. It can be seen that the critical current density $\left(J_{\mathrm{c}}\right)$ at zero field is $10,500 \mathrm{~A} / \mathrm{cm}^{2}$ and decreases with applied magnetic field, which is as expected for bulk YBCO.

It is possible to envisage that by making the initial preform more mechanically rigid, the multi-seeding technique could be refined to produce better growth and reduce the multi-grain nature of the resulting bulk superconductor. The infiltration growth (IG) technique [22, 28-32], which involves an infiltration of $\mathrm{Ba}-\mathrm{Cu}-\mathrm{O}$ rich liquid phase into a $\mathrm{Y}_{2} \mathrm{BaCuO}_{5}(\mathrm{Y}-211)$ preform at high temperatures and a subsequent reaction of Y-211 with the liquid to form the Y-123 superconducting phase during the slow cooling (SC) process, would be more advantageous for this kind of work. The advantage of the IG approach is that the sample undergoes a negligible shrinkage [31-33] during processing and simultaneously supports microstructures that contain fewer pores and cracks. Additionally, the IG technique often results in the formation of fine-sized spherical Y-211 inclusions in

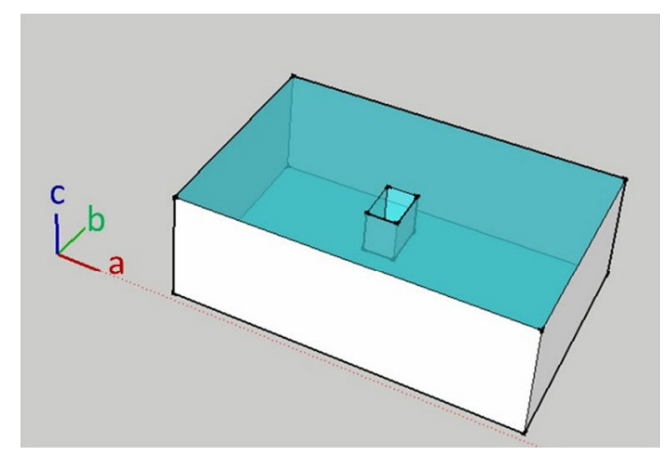

Fig. 7 Schematic illustration of the position of the small specimen in the parent single grain used to measure the temperature and field dependences of magnetization in order to estimate $T_{\mathrm{c}}$ and $J_{\mathrm{c}}$ 

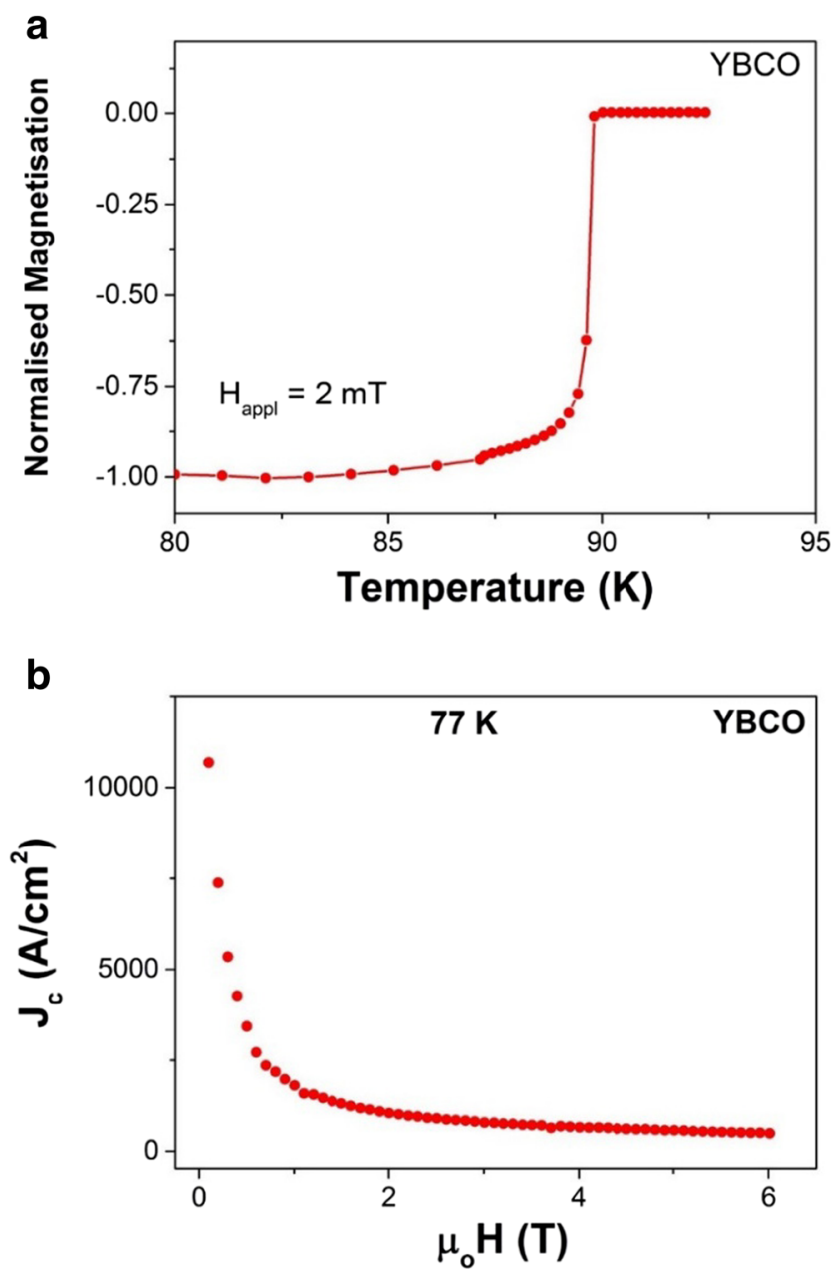

Fig. 8 a Temperature dependence of magnetization and $\mathbf{b}$ field dependence of the critical current density $\left(J_{\mathrm{c}}\right)$ obtained in the single-seeded YBCO sample

the Y-123 matrix, which is encouraging from a flux pinning point of view. The fact that the preform is stable during processing in IG makes it an efficient approach for multiseeding and for inhibiting misalignment of seed orientation at elevated processing temperature. Such a study is currently under investigation and will be reported in due course.

\section{Conclusion}

The bar-shaped, single-seeded and four-seeded samples of bulk YBCO were fabricated successfully via the TSMG technique, and the properties of the samples were measured and discussed. The measured trapped field values (on the surface of the samples) for the single-seeded sample and the four-seeded sample were 373 and $253 \mathrm{mT}$, respectively. The trapped field value for the single-seed sample is significantly higher than that of the four-seeded sample. Although there is a considerable difference in trapped field performance, the levitation force-displacement data were very similar for both samples. The transition temperature $\left(T_{\mathrm{c}}\right)$ and the critical current density at zero field $\left(J_{\mathrm{c}}(0)\right)$ of the single-seeded YBCO sample were observed to be $90 \mathrm{~K}$ and $10,500 \mathrm{~A} / \mathrm{cm}^{2}$ at $77 \mathrm{~K}$, respectively. It was observed from the microstructure of the four-seeded sample that the alignment of four seeds and growth profile was such that nucleation did not occur from the middle two seeds. We anticipate that by improving the alignment of the seeds and making the preform pellet mechanically rigid, the grains can be better aligned during melt processing to aid primarily the trapped field performance of the multi-seeded sample.

Acknowledgments This work was supported by the King Abdulaziz City for Science and Technology (KACST). Additional data related to this publication is available at the University of Cambridge data repository https://doi.org/10.17863/CAM.6785. All other data accompanying this publication are available directly within the publication.

Open Access This article is distributed under the terms of the Creative Commons Attribution 4.0 International License (http:// creativecommons.org/licenses/by/4.0/), which permits unrestricted use, distribution, and reproduction in any medium, provided you give appropriate credit to the original author(s) and the source, provide a link to the Creative Commons license, and indicate if changes were made.

\section{References}

1. Werfel, F.N.: Bulk materials. In: Seidel, P. (ed.) Applied superconductivity: handbook on devices and applications, p. 217. Wiley (2015). ISBN: 3527670661,9783527670666

2. Campbell, A.M., Cardwell, D.A.: Bulk high temperature superconductors for magnet applications. Cryogenics 37, 567-575 (1997)

3. Durrell, J.H. et al.: A trapped field of $17.6 \mathrm{~T}$ in melt-processed, bulk $\mathrm{Gd}-\mathrm{Ba}-\mathrm{Cu}-\mathrm{O}$ reinforced with shrink-fit steel. Supercond. Sci. Technol. 27, 082001 (2014)

4. Koshizuka, N., Tajima, S.: Advances in superconductivity XI. Springer Science and Business Media, 725-728 (2013)

5. Ikuta, H.: Melt processed RE-Ba-Cu-O bulk superconductors. In: Narlikar, A.V. (ed.) High temperature superconductivity 1: materials (2004). Spring Science and Business Media, New York, ISBN: 3-540-403631-X, pp. 79-108

6. Chow, J.C.L., Fung, P.C.W.: Fabrication of melt-texture growth YBCO samples using a simple muffle furnace. J. Supercond. 6, 365-372 (1993)

7. Yamaguchi, K., Murakami, M., Fujimoto, H., Kozhizuka, N., Tanaka, S.: Microstructures of $\mathrm{YBaCuO}$ fabricated by meltpowder-melt-growth process. Phys. C 185-189, 2497-2498 (1991)

8. Choi, C.H., Kim, H.S., Standard, O.C., Kim, M.J., Zhao, Y., Sorrell, C.C.: Powder melting process for $\mathrm{YBa}_{2} \mathrm{Cu}_{3} \mathrm{O}_{7-y}$ superconductor by eutectic formation. Supercond. Sci. Technol. 11, 637-644 (1998)

9. Cardwell, D.A., Hari Babu, N., Shi, Y.H., Iida, K.: Single grain (LRE)-Ba-Cu-O superconductors fabricated top seeded melt growth in air. J. Phys.: Conf. Ser. 43, 421-424 (2006) 
10. Nariki, S., Sakai, N., Murakami, M., Hirabayashi, I.: Processing of high-performance (Gd, Y)-Ba-Cu-O bulk superconductors with fine RE-211 pinning centers. IEEE Trans. Appl. Supercond. 15, 3110 (2005)

11. Meslin, S., Iida, K., Babu, N.H., Cardwell, D.A., Noudem, J.G.: The effect of Y-211 precursor particle size on the microstructure and properties of $\mathrm{Y}-\mathrm{Ba}-\mathrm{Cu}-\mathrm{O}$ bulk superconductors fabricated by seeded infiltration and growth. Supercond. Sci. Technol. 19, 711718 (2006)

12. Shi, Y.-H., Yoeh, W., Dennis, A.R., Hari Babu, N., Pathak, S., Xu, Z., Cardwell, D.A.: Growth rate of YBCO single grains containing Y-2411(M). J. Phys. Conf. Ser. 234, 012039 (2010)

13. Yang, W., Guo, X., Wan, F., Li, G.: Real-time observation and analysis of single-domain YBCO bulk superconductor by TSIG process. Cryst. Growth Des. 11, 3056-3059 (2011)

14. Zhai, W., Shi, Y.H., Durrell, J.H., Dennis, A.R., Rutter, N.A., Troughton, S.C., Speller, S.C., Cardwell, D.A.: The processing and properties of single grain $\mathrm{Y}-\mathrm{Ba}-\mathrm{Cu}-\mathrm{O}$ fabricated from graded precursor powders. Supercond. Sci. Technol. 26, 125021 (2013)

15. Ozturk, K., Sahin, E., Abdioglu, M., Kabaer, M., Celik, S., Yanmaz, E., Kucukomeroglu, T.: Comparative study of the magnetic stiffness, levitation and guidance force properties of single and multi-seeded YBCOs for different HTS-PMG arrangements. J. Alloys Compnd. 643, 201-206 (2015)

16. Shi, Y.-H., Durrell, J.H., Dennis, A.R., Cardwell, D.A.: Bulk YBCO seeded with 45-45bridge-seeds of different lengths. Supercond. Sci. Technol. 26, 015012 (2013)

17. Shi, Y., Durrell, J.H., Dennis, A.R., Zhang, Z., Zhai, W., Babu, N.H., Cardwell, DA.: A comparison of 0-0and 45-45bridge seeded, YBCO single grains. J. Amer. Ceram. Soc. 96, 1757-1762 (2013)

18. Cheng, L., Guo, L.S., Wu, Y.S., Yao, X., Cardwell, D.A.: Multiseeded growth of melt processed $\mathrm{Gd}-\mathrm{Ba}-\mathrm{Cu}-\mathrm{O}$ bulk superconductors using different arrangements of thin film seeds. J. Cryst. Growth 366, 1-7 (2013)

19. Li, T.Y., Wang, C.L., Sun, L.J., Yan, S.B., Cheng, L., Yao, X.: Multi-seeded melt growth (MSMG) of bulk Y-Ba-Cu-O using thin-film seeds. J. Appl. Phys. 108, 023914 (2010)

20. Yang, C.-M., Wang, S.-Y., Yi, C.H., Chen, P.-W., Chen, I.-G., $\mathrm{Wu}, \mathrm{M} .-\mathrm{K}$. : The optimal growth of single grain bulk $\mathrm{Y}-\mathrm{Ba}-\mathrm{Cu}-\mathrm{O}$ superconductors with $\mathrm{Nd}-\mathrm{Ba}-\mathrm{Cu}-\mathrm{O}$ thin film seed. IEEE Trans. Appl. Supercond. 23, 6800204 (2013)

21. Wongsatanawarid, A., Seki, H., Murakami, M.: Growth of large bulk $\mathrm{Y}-\mathrm{Ba}-\mathrm{Cu}-\mathrm{O}$ with multi-seeding. Supercond. Sci. Technol. 23, 045022 (2010)
22. Namburi, D.K., Shi, Y.-H., Palmer, K.G., Dennis, A.R., Durrell, J.H., Cardwell, D.A.: A novel, two-step top seeded infiltration and growth process for the fabrication of single grain, bulk (RE)BCO superconductors. Supercond. Sci. Technol. 29, 095010

23. Li, T., Cheng, L., Yan, S.B., Sun, L.J., Yao, X., Yoshida, Y., Ikuta, H.: Growth and superconductivity of REBCO bulk processed by a seed/buffer layer/precursor construction. Supercond. Sci. Technol. 23, 125002 (2010)

24. Shi, Y.-H., Dennis, A.R., Cardwell, D.A.: A new seeding technique for the reliable fabrication of large, SmBCO single grains containing silver using top seeded melt growth. Supercond. Sci. Technol. 28, 035014 (2015)

25. Devendra Kumar, N., Shi, Y.-H., Zhai, W., Dennis, A.R., Durrell, J.H., Cardwell, D.A.: Buffer pellets for high yield, top-seeded melt growth of large grain $\mathrm{Y}-\mathrm{Ba}-\mathrm{Cu}-\mathrm{O}$ superconductors. Cryst. Growth Des. 15, 1472-80 (2015)

26. Shi, Y., Hari Babu, N., Iida, K., Cardwell, D.A.: Mg-doped Nd$\mathrm{Ba}-\mathrm{Cu}-\mathrm{O}$ generic seed crystals for the top-seeded melt growth of large-grain (rare earth)-Ba-Cu-O bulk superconductors. J. Mater. Res. 21, 1355-1362 (2006)

27. Chen, D.X., Goldfarb, R.B.: Kim model for magnetization of typeII superconductors. J. Appl. Phys. 66, 2489-500 (1989)

28. Hari Babu, N., Kambara, M., Smith, P.J., Cardwell, D.A., Shi, Y.H.: Fabrication of large single-grain $\mathrm{Y}-\mathrm{Ba}-\mathrm{Cu}-\mathrm{O}$ through infiltration and seeded growth processing. J. Mater. Res. 15, 12351238 (2000)

29. Namburi, D.K., Shi, Y.-H., Palmer, K.G., Dennis, A.R., Durrell, J.H., Cardwell, D.A.: An improved top seeded infiltration growth method for the fabrication of $\mathrm{Y}-\mathrm{Ba}-\mathrm{Cu}-\mathrm{O}$ bulk superconductors. J. Eur. Ceram. Soc. 36, 615-624 (2016)

30. Li, G., Li, D., Deng, J., Yang, W.: Effects of liquid source mass and slow-cooling time on the infiltration growth of single-grain $\mathrm{Y}$ $\mathrm{Ba}-\mathrm{Cu}-\mathrm{O}$ bulk superconductors. J. Alloys. Compd. 551, 318-322 (2013)

31. Devendra Kumar, N., Rajasekharan, T., Seshubai, V.: Preform optimization in infiltration growth process: an efficient method to improve the superconducting properties of $\mathrm{YBa}_{2} \mathrm{Cu}_{3} \mathrm{O}_{7-\delta}$. Phys. C 495, 55-65 (2013)

32. Cloots, R., Koutzarova, T., Mathieu, J.P., Ausloos, M.: From RE-211 to RE-123. How to control the final microstructure of superconducting single-domains. Supercond. Sci. Technol. 18, R9-23 (2005)

33. Devendra Kumar, N., Shi, Y.H., Cardwell, D.A.: Fabrication of bulk (RE)BCO superconductors by the infiltration and growth process: past, present and future. In: Superconductivity: applications today and tomorrow, Nova Science Publishers Inc., New York, ISBN: 978-1-63483-816-0, pp. 1-35 (2015) 